\title{
La actividad física regular disminuye el riesgo de cáncer de mama
}

Physical activity and the risk of breast cancer. Thune I. Brenn T. Lund E. Gaard M. N Engl J Med 1997; 336 :1269-1275

\section{Objetivo}

Evaluar la influencia de la actividad física sobre el riesgo de cáncer de mama.

\section{Diseño}

Estudio poblacional de cohortes con una mediana de seguimiento de 13,7 años.

\section{Lugar}

Tres condados de Noruega.

\section{Participantes}

25.624 mujeres entre 20 y 54 años al inicio del estudio que respondieron encuestas sobre salud y actividad física recreacional y laboral ( $83 \%$ de respuesta).

\section{Evaluación de factores de riesgo}

Las participantes respondieron 2 encuestas (separadas por 3 a 5 años) sobre datos generales de salud, y dieta y fueron categorizadas en 4 grupos en base al grado de actividad física que desarrollaban tanto en su tiempo libre como en el trabajo (sedentaria, leve, moderada e intensa). Datos como peso, altura, tensión arterial, colesterol, etc. se obtuvieron durante el examen físico al inicio del estudio.

\section{Medición de resultados principales}

Todos los casos de cáncer de mama (351) fueron iden- tificados a través del registro nacional de cáncer de Noruega y un $98 \%$ verificados histologicamente. Se excluyeron las pacientes que desarrollaron cáncer o murieron durante el primer año del estudio.

\section{Resultados principales}

Ajustando por el efecto de la edad, obesidad y paridad, el Riesgo Relativo (RR) de cáncer de mama de las mujeres que realizaban ejercicio fue de 0,63 [IC 95\% $0,42$ a 0,95$]$. Esta reducción fue más importante en las premenopáusicas, RR 0,38 [IC 95\% 0,19 a 0,79]. En análisis estratificado, las mujeres no obesas que ejercitaban al menos $4 \mathrm{hs}$. por semana tuvieron la mayor reducción del riesgo $\mathrm{RR}$ 0,28 [IC 95\% 0,11 a 0,70]. Al comparar con el grupo basal (sedentarias), las distintas categorías de actividad física tanto en el tiempo libre como en el trabajo demostraron una disminución progresiva del riesgo de cáncer de mama.

\section{Conclusion}

La actividad física regular se asoció a la reducción del riesgo de cáncer de mama y este efecto fue más notable en las mujeres premenopáusicas.

\section{Comentario}

Más allá de los beneficios generales ya conocidos atribuídos a la actividad física, estos dos estudios demuestran una marcada reducción del riesgo de cáncer de mama y de la mortalidad global en mujeres que hacen ejercicio regularmente. En el primero, el efecto protector fue más notable en mujeres premenopáusicas que desarrollaban actividad física regular en forma habitual y en postmenopáusicas no obesas pero no demostró beneficio en el subgrupo total de postmenopáusicas, en quienes el riesgo de desarrollar cáncer de mama se incrementa notablemente. Los autores destacan que sus resultados son diferentes a un estudio que no encontró asociación entre ejercicio y riesgo de ca de mama porque la mayoría de las participantes eran postmenopáusicas. Si bien el estudio reporta los resultados en términos de $R R$ y reducción de $R R$, lamentablemente no provee el número de mujeres pre y postmenopáusicas para poder calcular los riesgos absolutos de cáncer de mama en cada grupo y estimar el impacto global del beneficio atribuible al ejercicio. Los autores especulan con que la actividad física sostenida puede disminuir la secreción de estrógenos y progesterona y, por lo tanto, disminuir a largo plazo la exposición acumulativa de la glándula a la acción carcinogenética de estas hormonạs. El segundo estudio ilustra claramente la relación inversa que existe entre el ejercicio regular y la mortalidad global en mujeres postmenopáusicas, y destaca la consistencia de este hallazgo con el de otros estudios de similar diseño pero de menor tamaño. Este estudio evaluó el grado de actividad de las participantes solo al inicio, sin tomar en cuenta la duración ni las eventuales modificaciones que pudieron haber ocurrido en el tiempo mientras que en el estudio Noruego la categorización resultó de la combinación de 2 encuestas separadas por 3 a 5 años. Al analizar por causas de muerte, las reducciones más importantes fueron las debidas a causas cardiovasculares y respiratorias, coincidente con otros estudios realizados en hombres, pero no con el de Framingham, en el que no se demostró asociación entre ejercicio y mortalidad cardiovascular luego de 16 años de seguimiento. Las discrepancias entre los distintos estudios pueden deberse no sólo a los distintos métodos utilizados para medir el grado de ac- 